Distinguishing Sensory Sensitivity and Reactivity, and how they relate to Autistic Traits

Schulz, S. E. ${ }^{1,2}$ \& Stevenson, R. A. ${ }^{1,2}$

1. Brain and Mind Institute, Western University

2. Department of Psychology, Western University 


\begin{abstract}
Sensory processing issues are common across neurodevelopmental disorders, including attention-deficit/hyperactivity disorder, obsessive compulsive disorder and intellectual disabilities, as well as other mental health disorders such as schizophrenia, anxiety, and depression. This study uses a novel behavioural paradigm and a questionnaire to assess sensory issues and these two methods are directly compared to distinguish sensory sensitivity and sensory reactivity. We also used autistic traits as an empirical testbed to shed light on the relationships between sensory processing issues and traits associated with neurodevelopmental disorders. Sensory processing issues are highly prevalent in the autistic population, and previous findings have strongly supported a relationship between parent or self-reported sensory sensitivity and autistic traits, whereas studies that have examined this relationship through behavioural assessments of sensitivity are less consistent. The current study explores these differences and suggests that sensory sensitivity and sensory reactivity are distinct constructs, with questionnaires assessing reactivity whereas behavioural measures assess sensitivity. One hundred and eighteen typically-developed adults completed a visual detection task, an auditory detection task, and questionnaires on sensory processing and autistic traits. Visual thresholds, derived from the behavioural paradigm and self-report visual sensitivity were not correlated, but both were related to and predictive of autistic traits. Auditory thresholds and self-report auditory sensitivity were also unrelated. Overall, sensitivity is highly associated with autistic traits, however, behavioural and questionnaire assessments of sensitivity lack convergent validity and, therefore, likely assess distinct constructs. In conclusion, sensory sensitivity and sensory reactivity are unique concepts that fall under the umbrella of sensory processing differences and need to be researched as such in relation to behavioural traits across clinical populations.
\end{abstract}




\section{Distinguishing Sensory Sensitivity and Reactivity, and how they relate to Autistic Traits}

Sensory processing difficulties are common among most developmental disorders and mental health concerns, including attention-deficit/hyperactivity disorder (Ghanizadeh, 2011), intellectual disabilities (Engel-Yeger, Hardal-Nasser, \& Gal, 2011), schizophrenia (Javitt, 2009), depression (Serafini et al., 2017), obsessive-compulsive disorder, and anxiety (Houghton, Stein, \& Cortese, 2020), among others. Sensory processing difficulties in these populations have been linked to higher-order clinical and behavioural symptoms (Elliott, Romer, Knodt, \& Hariri, 2018; Piek \& Dyck, 2004; Wallace \& Stevenson, 2014), findings that have led to an increased focus on this burgeoning area of research. With that said, there is still considerable debate in the field on how sensory processing difficulties should be conceptualized and measured within these populations. One particular clinical population in which sensory issues have been widely studied is autism spectrum disorder (ASD). Studying the relationships between sensory processing and behavioural traits in ASD provides an optimal platform for this line of work due to the high prevalence and severity of sensory processing issues observed in this population (Rogers \& Ozonoff, 2005). In ASD, difficulties in sensory processing span all sensory modalities and encapsulate a wide range of perceptual difficulties (Ashwin, Ashwin, Rhydderch, Howells, \& Baron-Cohen, 2009; Baum, Stevenson, \& Wallace, 2015; Bertone, Mottron, Jelenic, \& Faubert, 2003; Blakemore et al., 2006; Bonnel et al., 2010; Caplette, Wicker, \& Gosselin, 2016; Clery et al., 2013; Hrdlicka et al., 2011; Jones et al., 2009; Khalfa et al., 2004; Kinsbourne, 1980; Marco, Hinkley, Hill, \& Nagarajan, 2011; McCleery, Allman, Carver, \& Dobkins, 2007; Simmons et al., 2009). Sensory sensitivity, specifically, is linked to autistic traits and in many cases has been used to discriminate between autistic and neurotypical individuals. Many studies have indicated that sensory 
sensitivity is strongly correlated with, and even predictive of, autistic traits and symptoms. (Baranek, David, Poe, Stone, \& Watson, 2006; Crane, Goddard, \& Pring, 2009; Kern et al., 2006; Mayer, 2017; Robertson \& Simmons, 2013; Schulz \& Stevenson, 2018; Talay-Ongan \& Wood, 2000; Tomchek \& Dunn, 2007; Watling, Deitz, \& White, 2001). These findings support the hypothesis that sensory sensitivity plays an important role in the pattern of symptoms observed in ASD. However, much of the research on sensory sensitivity has utilized parentor self-report questionnaires to assess sensory sensitivity, but these questionnaires have their limitations. Namely, the accuracy of these questionnaires requires insight into complex internal sensory processes from the individual or their parents. Thus, objective, behavioural measures of sensory sensitivity may be a more appropriate assessment tool to address whether increased sensory sensitivity is related to an increase in autistic traits.

Previous studies that have analyzed the relationship between behaviourally-assessed sensory sensitivity and autistic traits have demonstrated inconclusive results compared to the well-established questionnaire literature. Visual and auditory processing are the most commonly-studied sensory domains in ASD, and the studies that investigate the relative impairment in ASD within these domains have displayed mixed findings (Haesen, Boets, \& Wagemans, 2011; Kwakye, Foss-Feig, Cascio, Stone, \& Wallace, 2011; O'connor, 2012; Simmons et al., 2009). For example, studies have shown that visual sensitivity is heightened in autistic individuals compared to neurotypical controls, such that visual acuity (Ashwin et al., 2009) and contrast sensitivity when using simple stimuli (Bertone, Mottron, Jelenic, \& Faubert, 2005) are greater in autistic participants compared to controls. Research has also shown that detection thresholds decrease in association with increases in autistic traits, which supports the relationship between autistic traits and visual sensitivity even in the neurotypical population (Schulz \& Stevenson, 2019). However, additional studies have used similar 
methods and have found opposing results such that no differences exist between ASD and neurotypical groups in visual acuity (Tavassoli, Latham, Bach, Dakin, \& Baron-Cohen, 2011), contrast sensitivity (De Jonge et al., 2007), or detection thresholds (Behrmann et al., 2006). In conclusion, regardless of task type, the question remains as to whether autistic individuals exhibit greater sensitivity to visual stimuli compared to neurotypical individuals.

In ASD, the evidence in support of greater sensitivity in the auditory domain is more consistent than in the visual domain, but there are still discrepant findings within the audition literature. For example, increased pitch sensitivity (Bonnel et al., 2003), pitch discrimination (Bonnel et al., 2003; Stewart, Griffiths, \& Grube, 2018), distress to noise (Bonnel et al., 2003), and loudness perception (Khalfa et al., 2004) have all been reported in ASD compared to their neurotypical peers. Alternatively, the same study that showed increased distress to noise in autistic individuals also found no difference in auditory thresholds between autistic participants and controls (Khalfa et al., 2004). Additionally, numerous studies have found differences in auditory perception but only in a subgroup of the autistic population who display the most severe range of autistic symptoms or language delays (Bonnel et al., 2010; Jones et al., 2009; O'connor, 2012). In fact, no differences were found between autistic adults with a high IQ (mean IQ = 114.8) and neurotypical controls in discrimination thresholds or samedifference auditory judgments (Haigh et al., 2016). Therefore, it is unclear whether differences in auditory perception are observable at varying levels across the entire autism spectrum or only in autistic individuals expressing certain traits or a certain level of severity within specific traits. Furthermore, there have been inconsistent findings in relation to stimulus complexity, and one study suggested that autistic individuals exhibit enhanced auditory sensitivity but only when the simplest stimuli are used (Järvinen-Pasley, Wallace, Ramus, Happé, \& Heaton, 2008). Thus, perceptual differences are apparent in the auditory domain in some autistic 
individuals, however, these differences, which may be interpreted as either enhancements or deficits, may be dependent on factors such as stimulus type and/or the patterns of autistic traits expressed.

While the literature has been inconsistent regarding the relationships between sensory processing and autistic traits and symptoms between the various sensory modalities, these results have also varied across measurement type. (Baranek et al., 2006; Crane et al., 2009; Kern et al., 2006; Mayer, 2017; Robertson \& Simmons, 2013; Schulz \& Stevenson, 2018; Talay-Ongan \& Wood, 2000; Tomchek \& Dunn, 2007; Watling et al., 2001). Sensitivity differences between autistic and neurotypical individuals have been observed across multiple questionnaires including the Sensory Profile (Dunn, 1999), the Sensory Experiences Questionnaire (Baranek et al., 2006), the Glasgow Sensory Questionnaire (Robertson \& Simmons, 2013), and the Sensory Sensitivity Questionnaire - Revised (Talay-Ongan \& Wood, 2000). Regardless of the questionnaire measure used, greater sensory sensitivity has been found in autistic populations of all ages when compared to neurotypical peers. These differences encompass the full range of sensory modalities, including vision, audition, touch, gustatory, olfactory, proprioceptive, and vestibular. Additionally, the literature has demonstrated that typically-developing individuals who display greater autistic traits also exhibit increased sensory sensitivities, which provides evidence for the positive relationship between sensitivity and autistic traits across the entire spectrum from typically-developing to clinically-diagnosable ASD (Robertson \& Simmons, 2013; Schulz \& Stevenson, 2018). Taken together, the evidence from studies that have utilized questionnaires unequivocally supports the relationship between sensory sensitivity and autistic traits despite the inconclusive findings stemming from the studies that have utilized behavioural measures of sensory sensitivity. 
The question remains as to why questionnaire data has provided unambiguous support for the relationship between sensory sensitivity and autistic traits and behavioural results display less consistent findings. As far as we are aware, only one study has directly compared questionnaire and behavioural measures of sensory sensitivity in audition or vision in relation to autistic traits or symptoms (Schulz \& Stevenson, 2019). This study displayed that visual thresholds, assessed through a behavioural psychophysics paradigm using the method of constant stimuli, were associated with autistic traits such that increased sensitivity was related to more frequent and more severe restricted interests and repetitive behaviours. Self-report data on sensory sensitivity was collected from the same individuals using the Sensory Profile 2, which was also strongly related to restricted interests and repetitive behaviours. Finally, and perhaps most interestingly, the two assessments of sensory sensitivity were unrelated to one another.

It has been theorized that self-reported measures of sensory sensitivity actually assess sensory reactivity, or the individuals' reactions to sensory input, whereas behavioural tasks are a more precise assessment of sensory sensitivity (Schulz \& Stevenson, 2019). This distinction has been hypothesized as the self-report questionnaire includes the following items to describe sensitivity: "I gag easily with food textures or food utensils.", "I startle easily from unexpected or loud noises.", "I find it difficult to work with a lot of background noise.", and "I am uncomfortable with certain fabrics." The problem is that many of these examples refer to the behavioural response (for example, to gag or to startle) to a sensory stimulus opposed to the perception of that stimulus. Whereas a behavioural detection task objectively determines whether some individuals can detect stimuli at a lower intensity than others, suggesting greater sensitivity to that stimuli. 
In a study of loudness perception, no differences were found in the auditory thresholds of autistic individuals compared to neurotypical individuals even though the same autistic individuals subjectively rated noises as louder than their neurotypical counterparts (Khalfa et al., 2004). In the typically-developed population, a meta-analysis of eleven studies has examined acoustic reflex thresholds and levels of auditory discomfort. The findings suggested that auditory discomfort cannot be predicted based on acoustic reflex thresholds in individuals with normal hearing (Olsen, 1999). Taken together, these results further support the hypothesis that sensory thresholds, automatic responses to stimuli, and subjective perception of sensory stimuli may differ in both the general and autistic populations.

The current study aimed to further investigate this theory, and to explore the idea that questionnaires assess sensory reactivity, while behaviour measures assess sensory sensitivity. By including both visual and auditory behavioural measures and a broad range of autistic traits, this study allowed for a detailed examination of the relationship between sensory processing and behavioural traits. To this end, we administered psychophysical tasks to assess sensory sensitivity, as well as sensory questionnaires to directly compare the two assessment methods. We predicted that behavioural thresholds for auditory and visual stimuli would not be related to the sensitivity quadrant of the sensory questionnaires, as previously reported (Schulz \& Stevenson, 2019). Secondly, this study aimed to determine whether autistic traits were related to sensory sensitivity and/or sensory reactivity in a neurotypical population. Relationships between both the results of the psychophysical tasks and sensory questionnaires and both arms of the ASD diagnostic criteria, restricted interests and repetitive behaviours and social and communication deficits, were expected such that as sensitivity increased, so to does the incidence of autistic traits.

\section{Methods}




\section{Participants}

One hundred and thirty participants were recruited from a university online volunteer system. Participants were native-English speaking and had self-reported normal or correctedto-normal vision and normal hearing. Participants were excluded if they failed to reach both a visual and auditory threshold throughout the experiment $(\mathrm{N}=12)$, which resulted in a final sample of 118 individuals. The average age of participants was 18.5 (SD $=1.70$; range: 17 to 29) years of age and 78 self-reported gender as female, 39 male, and 1 other. Participants were recruited based upon the Research Diagnostic Criteria (RDoC) framework (Cuthbert \& Insel, 2013; Insel et al., 2010) which relies on studying the mechanisms of dimensional constructs of symptomology in all individuals. As such, participants were not included or excluded based on presence or absence of any diagnoses.

\section{Overview}

Participants completed an adaptive-staircase detection task in both the auditory and visual domains. Participants also completed a battery of questionnaires including the Broad Autism Phenotype Questionnaire (BAPQ; Hurley, Losh, Parlier, Reznick, \& Piven, 2007) and the Adolescent/Adult Sensory Profile (AASP; Brown \& Dunn, 2002). Participants provided written consent and all aspects of this project were approved by a university non-medical ethics board.

\section{Stimuli}

All stimuli were presented using E-Prime 3 (Psychology Software Tools, 2016) software with Tobii Pro Extensions (Tobii, 2018) on a monitor with a refresh rate of $16.67 \mathrm{~ms}$ $(60 \mathrm{~Hz})$. The visual stimuli consisted of 70 sinusoidal luminance gratings, or Gabor patches, that varied in Michelson contrast from 0.01 to 0.1 , evenly spaced (see Figure 1 for an 
example of the visual stimuli). Visual stimuli subtended $9^{\circ}$ of visual angle, with a cycle frequency of 30 cycles per degree (Kéita, Guy, Berthiaume, Mottron, \& Bertone, 2014). All visual stimuli were embedded in static visual noise. Every visual trial that presented the same contrast intensity was also presented at the same orientation which was randomly selected from the possible range of 30 to 60 or -30 to $-60^{\circ}$ for each different contrast intensity. Comparably, the auditory stimuli consisted of 70 pure tones presented at every $1 / 2$ decibel from 35 to $69.5 \mathrm{~dB}$ SPL. Auditory tones were presented at a frequency of $800 \mathrm{~Hz}$ embedded in continuous $40 \mathrm{~dB}$ SPL auditory white noise. Null trials used the same visual and auditory noise without an embedded Gabor patch or auditory tone.

\section{Procedure}

Participants were seated in a dark room $60 \mathrm{~cm}$ away from the monitor (Acer LCD Monitor, model: X223W) of a desktop computer (Lenovo ThinkCentre M710s, model: 0037US). A Tobii Pro X3 - 120 eye-tracker was attached to the monitor for gaze-contingent trial control. A chin rest was used to minimize head motion and control the distance to the monitor, which was adjusted so the centre of the monitor was aligned with each participant's eye level. The experiment began with a 9-point calibration using the TETcallibration package call. Subsequently, the participant completed practice trials to become accustomed to the background light and noise and to familiarize themselves with the experimental stimuli. During the task, participants were instructed to indicate the presence of any stimulus, either auditory or visual, by pressing the space bar as quickly and as accurately as possible.

The task consisted of 600 trials divided into auditory (150), visual (150), and null trials (300), ordered semi-randomly, such that each block consisted of 3 auditory trials, 3 visual trials, and 6 null trails. Auditory and visual white noise were presented throughout the entire experiment, with no clear trial boundaries apparent to the participants. Each trial began with 
the continued presentation of auditory and visual noise for a minimum of $900 \mathrm{~ms}$. The program did not proceed until participants fixated to the screen for $200 \mathrm{~ms}$, carried out through gaze-contingent trial control. An auditory or visual stimulus was then presented in the continuous noise for $100 \mathrm{~ms}$, or in the case of a null trial, auditory and visual noise continued for $100 \mathrm{~ms}$, with no stimulus presentation. Subsequent to stimulus or null presentation, auditory and visual noise continued for up to $1500 \mathrm{~ms}$ during which possible responses were collected, before the next trial began, with no noticeable transition between trials. If the participant responded, the response period ended immediately, and the next trial began without a break in the visual and auditory noise.

Sensory thresholds were defined as the minimum detectable stimulus intensity for each participant, quantified as the stimulus intensity that was accurately detected by the participant in $50 \%$ of presentations. Trials were presented in six interleaved one-up-one-down adaptive staircases; three in the auditory domain and three in the visual domain. Thus, if a participant accurately detected a stimulus upon presentation, the stimulus presented in the subsequent trial within the same staircase was reduced in intensity (increased difficulty of detection). Alternatively, if a participant failed to detect a stimulus upon presentation, the stimulus presented in the subsequent trial within the same staircase was increased in intensity (decreased difficulty of detection). Each staircase increased or decreased in difficulty by 8 levels until the first reversal. A reversal occurs when a missed presentation follows an accurately detected presentation, or an accurate detection follows a missed presentation. The reversal implies a shift from the stimuli intensity increasing in difficulty from the previous trial to decreasing in difficulty in the subsequent trial, or vice versa. For example, in a staircase beginning with a low intensity stimulus (difficult to detect), the participant likely missed the first few stimuli presentations in a row as they are below the participant's detection threshold, and 


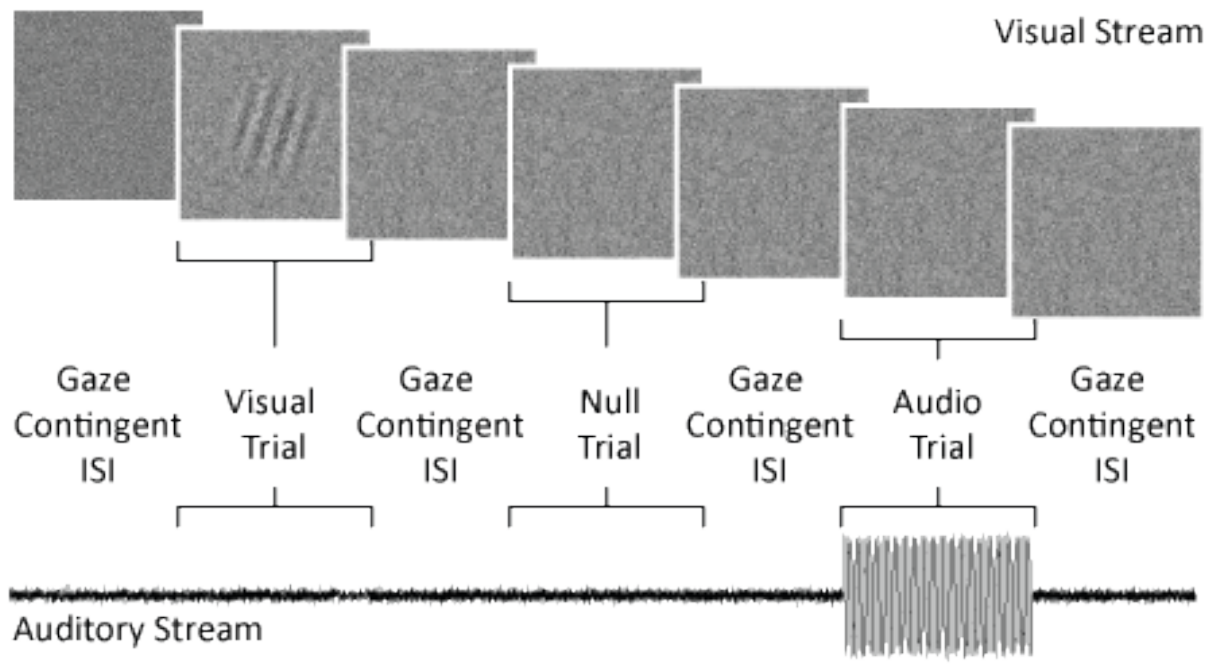

Figure 1: Trial procedure with example of visual and auditory stimuli.

with each missed trial, the intensity of the stimuli continued to increase (get easier) within the respective staircase. Once the staircase surpassed the participant's detection threshold, the participant detected the stimulus, the first reversal occurred, and the next stimulus was less intense (more difficult to detect). As the participant approached their threshold, reversals occurred on approximately $50 \%$ of the trials, such that the participant is presented with a stimulus they are just barely able to detect, followed by an undetectable stimulus. After the first reversal, step size was reduced to 4 levels until the second reversal. After the fourth reversal, step size was reduced to 2 levels, until the sixth reversal, at which point, step size was reduced to 1 step per trial.

In the visual domain, staircases began at a Michelson contrast of 0.017 (difficult), 0.056 (moderately difficult), and 0.095 (easy). Likewise, in the auditory modality, staircases began at 37.5 (difficult), 52.5 (moderately difficult), and 67.5 (easy) dB SPL. As the experiment progressed, the three staircases in each domain converged and a threshold was established as the median level of the three staircases after six reversals were made within each staircase in each sensory domain (see Figure 2). Thus, a threshold was determined 
independently in the auditory and visual domains and will be referred to as visual and auditory thresholds moving forward.

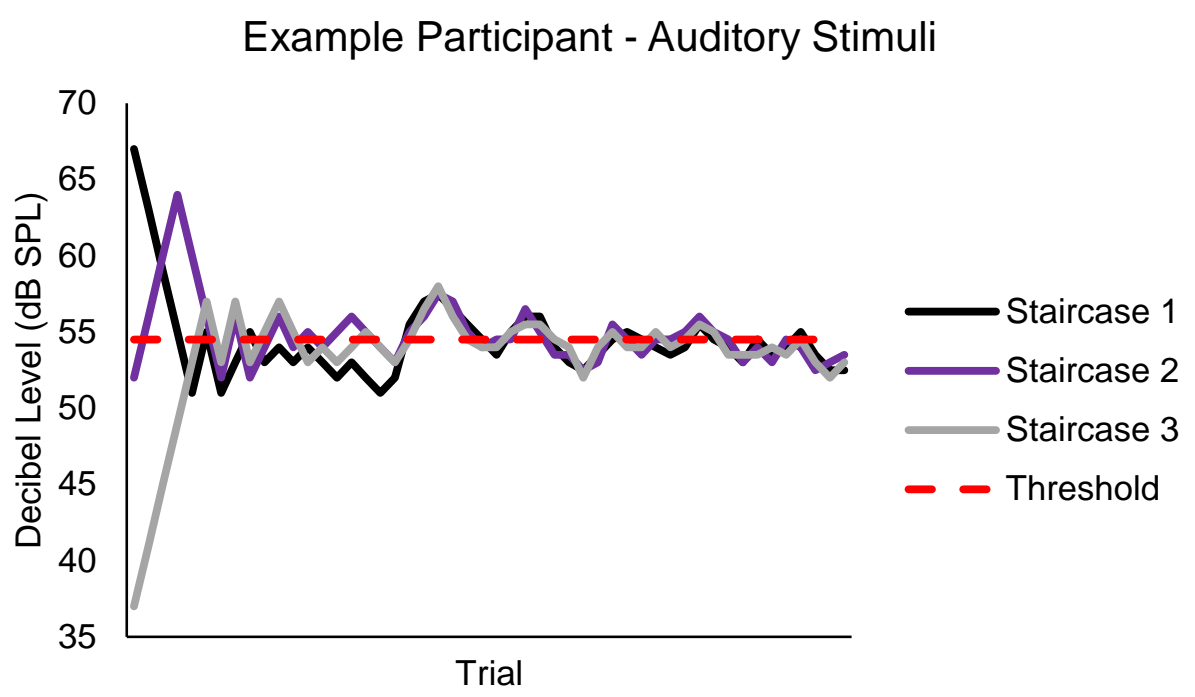

Figure 2: Individual participant data on auditory task.

\section{Questionnaires}

Broad Autism Phenotype Questionnaire. The BAPQ is an assessment of autistic traits designed for use in the general population (Hurley et al., 2007). Its subscales assess three aspects of the broader autism phenotype, including aloofness, rigidity, and pragmatic language problems. Scores on each subscale of the BAPQ can range from 1 to 6 . Someone with high levels of aloofness, according to the BAPQ, lacks a desire to participate in social interactions, whereas a rigid personality describes someone with difficulty adjusting to change. Finally, those with pragmatic language problems struggle to use language in a social context. These are all issues that are often observed in the autistic population with greater severity than can be found in the general population (Hurley et al., 2007). The BAPQ displays high internal consistency in each of the three subscales $(\alpha>.0 .85)$ and has strong sensitivity and specificity (> 70\%) (Hurley et al., 2007). 
Adolescent/Adult Sensory Profile. The AASP assesses four patterns of sensory processing, labelled hypersensitivity, low registration, sensory seeking, and sensory avoidance. These patterns are well documented in six different domains including taste/smell, touch, vision, audition, movement, and activity level (Brown \& Dunn, 2002). This 60-item, selfreport measure utilizes a five-point Likert scale. Possible scores range from one, indicating a behaviour that is never performed to a score of five in which the behaviour is almost always performed. The range of the auditory and visual sensitivity scales range from 0 to 15 . Example items include covering of the ears to avoid loud sounds, preferring to work in low lighting, and being an extremely picky eater. For the purpose of this study, we focused on the Visual and Auditory Hypersensitivity scales; we referred to these scales as self-report visual and auditory sensitivity.

The Sensory Profile questionnaires, including the AASP, are the most commonly used parent- and self-report tools to assess sensory processing in individuals with ASD and the various versions are very well validated. The item reliability for each of the four factors of the AASP specifically ranges from an $\alpha=0.66$ for the Sensory Avoiding quadrant to an $\alpha=0.82$ for the Low Registration quadrant; the four factor model demonstrates a good fit for sensory data and is also consistent with convergent data from skin-conductance tests in response to sensory stimuli (Brown, Tollefson, Dunn, Cromwell, \& Filion, 2001). Additionally, the AASP has been normed in adults with ASD (Crane et al., 2009).

\section{Analysis}

Kolmogorov-Smirnov normality tests were used to assess the normality of the data. Of the seven scales of interest, only the Aloof Personality subscale of the BAPQ was normally distributed, thus nonparametric tests were used. Spearman rank-order correlations were used to determine the relationships between self-report sensitivity and thresholds in both auditory 
and visual domains. Furthermore, Spearman rank-order correlations were conducted to analyze relationships between sensory processing and behavioural traits, thus resulting in a correlation matrix of autistic traits and each measure of visual and auditory sensitivity. Multiple comparisons were controlled for using the Benjamini-Hochberg method using a false discovery rate (FDR) of $Q=.15$ (Benjamini \& Hochberg, 1995). All p-values less than 0.05 survived correction according to FDR critical values and unadjusted $p$-values are reported below.

Post hoc regressions were conducted to determine whether auditory and visual thresholds and/or self-report auditory and visual sensitivity are predictive of autistic trait, where appropriate. Visual threshold and self-report visual sensitivity values were inputted as predictor variables into four regression analyses predicting the total score and each of the three subscales of the BAPQ.

\section{Results}

\section{Participants}

All participants' data were used in the analysis of the auditory thresholds and the average auditory threshold was $48.24 \mathrm{~dB} S P L(S D=3.13)$ ranging from 40.5 to $59 \mathrm{~dB} S P L$. Of the total sample, only 107 participants reached the visual threshold for inclusion and the average visual threshold of included participants was a Michaelson contrast of 0.05 (SD = $0.02)$, ranging from 0.02 to 0.1 . AASP visual reactivity averaged $5.68(S D=2.12)$, ranging from 1 to 13 , whereas AASP auditory reactivity was $8.71(S D=2.67)$ with a range of 1 to 15 . Average Total BAPQ scores ranged from 1.25 to $4.69(M=2.96, S D=0.58)$, Aloofness scale ranged from 1 to $5.17(M=2.94, S D=0.79)$, Pragmatic Language scale ranged from 1 to 4 $(M=2.81, S D=0.58)$, and Rigidity scale ranged from 1.16 to $5.08(M=3.13, S D=0.72)$. 


\section{Behavioural Thresholds and Self-Report Sensitivity}

To compare behavioural thresholds and self-report sensitivity, a correlational analysis was conducted between visual thresholds and self-report visual sensitivity and between auditory thresholds and self-report auditory sensitivity. Neither of these relationships were significant $\left(r_{s(105)}=0.098, p=0.314,95 \% \mathrm{Cl}:-0.084\right.$ to 0.288 and $r_{s(116)}=-0.009, p=0.926$, $95 \% \mathrm{Cl}:-0.192$ to 0.180 , respectively). Therefore, these two measures that were intended to assess the same construct, were not related (see Figure 3).
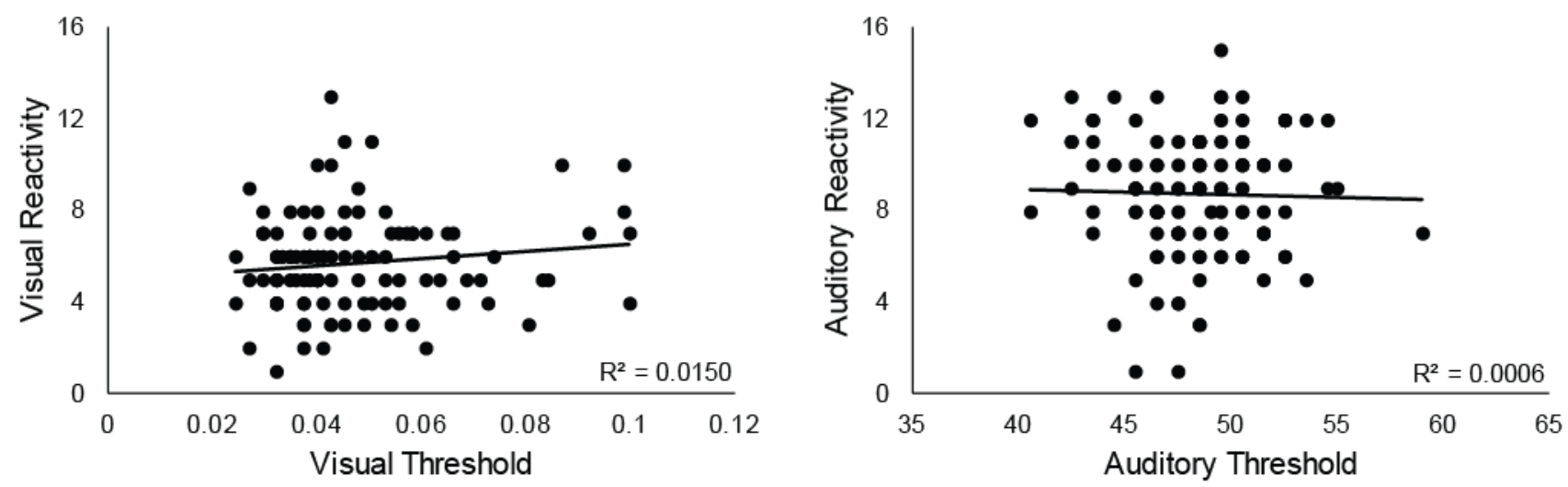

Figure 3: Correlations between behavioural thresholds and self-reported sensitivity in both the auditory and visual modalities.

\section{Behavioural Thresholds and Autistic Trait}

To examine the relationships between auditory and visual thresholds and autistic traits, Spearman rank-order correlations were conducted between each threshold and BAPQ subscale and total scores. Visual thresholds were significantly related to Total BAPQ scores $\left(r_{s(105)}=-0.203, p=0.036,95 \% \mathrm{Cl}:-0.371\right.$ to 0$)$, the Aloof Personality subscale $\left(r_{s(105)}=-\right.$ $0.244, \mathrm{p}=0.011,95 \% \mathrm{Cl}:-0.423$ to -0.034 ), and the correlation between visual thresholds and Rigid Personality was trending towards significance $\left(r_{s(105)}=-0.170, p=0.081,95 \% \mathrm{Cl}\right.$ : 0.342 to -0.017$)$. Finally, visual thresholds were not significantly related to the Pragmatic Language subscale $\left(r_{s(105)}=-0.028, p=0.778,95 \% \mathrm{Cl}:-0.205\right.$ to 0.156$)$. These data suggest 
that individuals with greater sensitivity to visual stimuli display greater autistic traits in general, and more specifically, have a greater tendency to be aloof and rigid (see Figure 4).
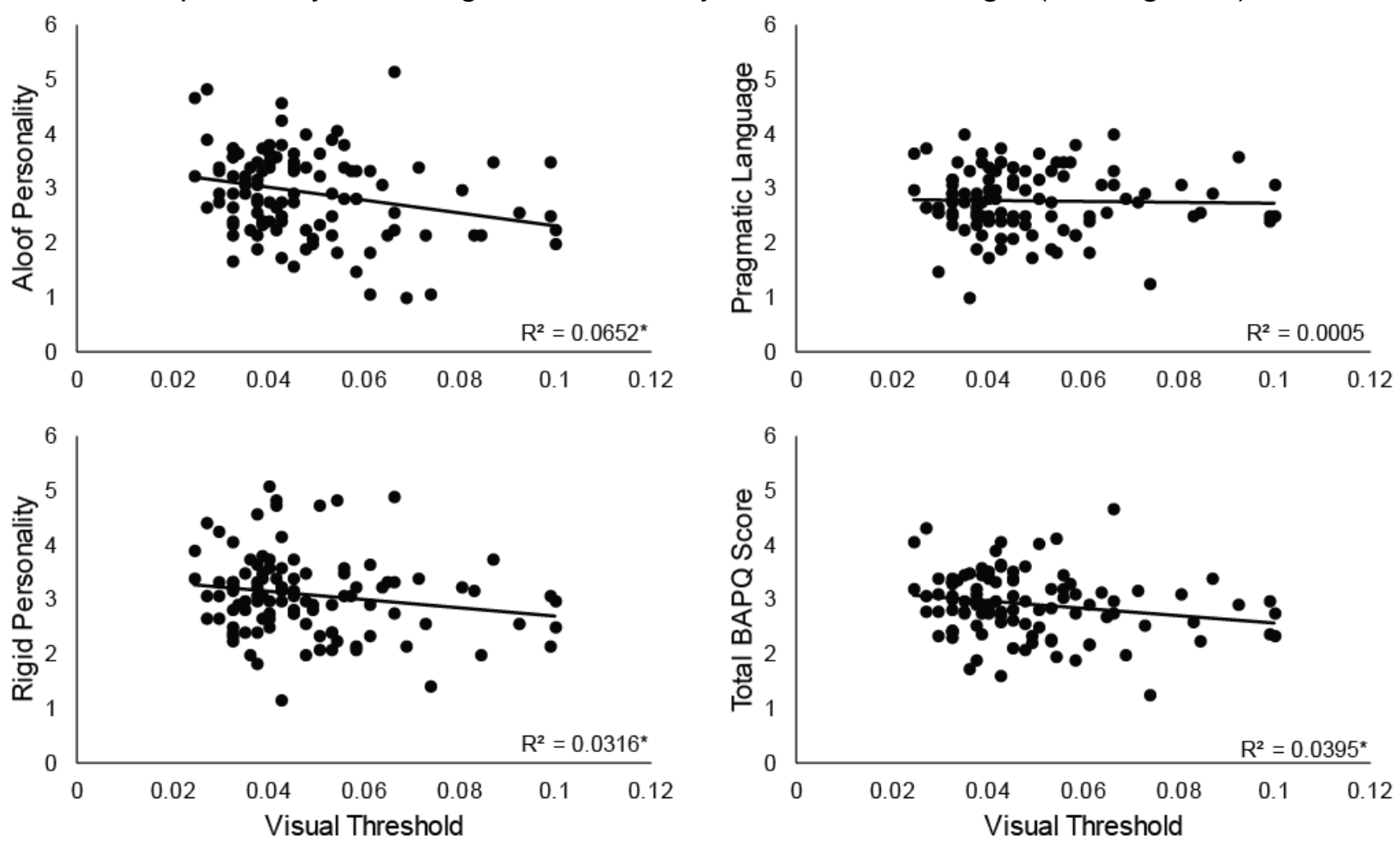

Figure 4: Correlations between visual thresholds and BAPQ subscales and Total Score.
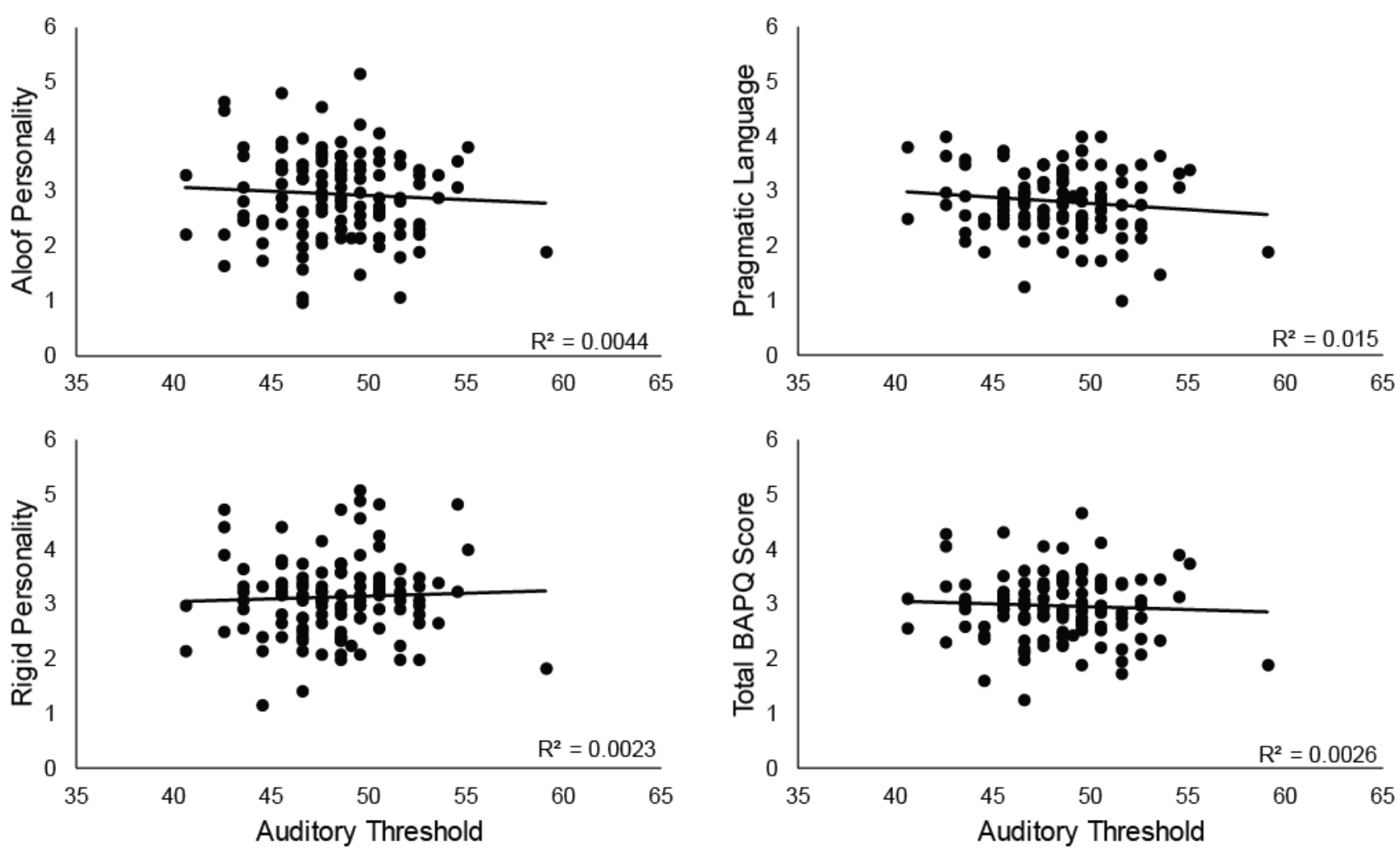

Figure 5: Correlations between auditory thresholds and BAPQ subscales and Total Score. 
Auditory thresholds were not significantly related to any of the BAPQ subscales (Aloof: $r_{s(116)}=-0.057, p=0.540,95 \% \mathrm{Cl}:-0.242$ to $0.133 ;$ Pragmatic: $r_{s(116)}=-0.066, p=0.481,95 \%$ $\mathrm{Cl}:-0.253$ to 0.137 ; Rigid: $r_{s}(116)=-0.066, p=0.445,95 \% \mathrm{Cl}:-0.119$ to 0.259$)$ or the Total BAPQ score $\left(\mathrm{r}_{\mathrm{s}(116)}=-0.022, \mathrm{p}=0.811,95 \% \mathrm{Cl}:-0.213\right.$ to 0.183$)$. Thus, there was no evidence for a relationship between auditory sensitivity and autistic traits as measured by the BAPQ (see Figure 5).

\section{Self-Report Sensitivity and Autistic Traits}

Self-report visual sensitivity was significantly correlated with Total BAPQ score $\left(\mathrm{r}_{\mathrm{s}(116)}=\right.$ $0.305, p=0.001,95 \% \mathrm{Cl}: 0.121$ to 0.468$)$, Aloof Personality $\left(r_{s(116)}=0.305, p=0.001,95 \%\right.$ $\mathrm{Cl}: 0.121$ to 0.464$)$, and Pragmatic Language $\left(\mathrm{r}_{\mathrm{s}(116)}=0.304, \mathrm{p}=0.001,95 \% \mathrm{Cl}: 0.126\right.$ to 0.459), but not Rigid Personality $\left(r_{s(116)}=0.128, p=0.167,95 \% \mathrm{Cl}:-0.059\right.$ to 0.294$)$. Therefore, self-report visual sensitivity was highly related to a lack of interest in social interaction and resistance to change, but not the ability to maneuver the social aspects of language (see Figure 6).

Unlike the auditory thresholds, self-report auditory sensitivity was significantly correlated to all aspects of the broader autism phenotype including Aloof Personality $\left(r_{s(116)}=\right.$ $0.196, p=0.003,95 \% \mathrm{Cl}: 0.014$ to 0.369$)$, Rigid Personality $\left(r_{s(116)}=0.202, p=0.028,95 \%\right.$ $\mathrm{Cl}: 0.018$ to 0.370$)$, Pragmatic Language $\left(r_{s(116)}=0.320, p<0.001,95 \% \mathrm{Cl}: 0.149\right.$ to 0.473$)$, and Total BAPQ score $\left(r_{s(116)}=0.302, p=0.001,95 \% \mathrm{Cl}: 0.128\right.$ to 0.450$)$. Thus, self-report auditory sensitivity was very highly related to the broader autism phenotype in all measured aspects (see Figure 7). 

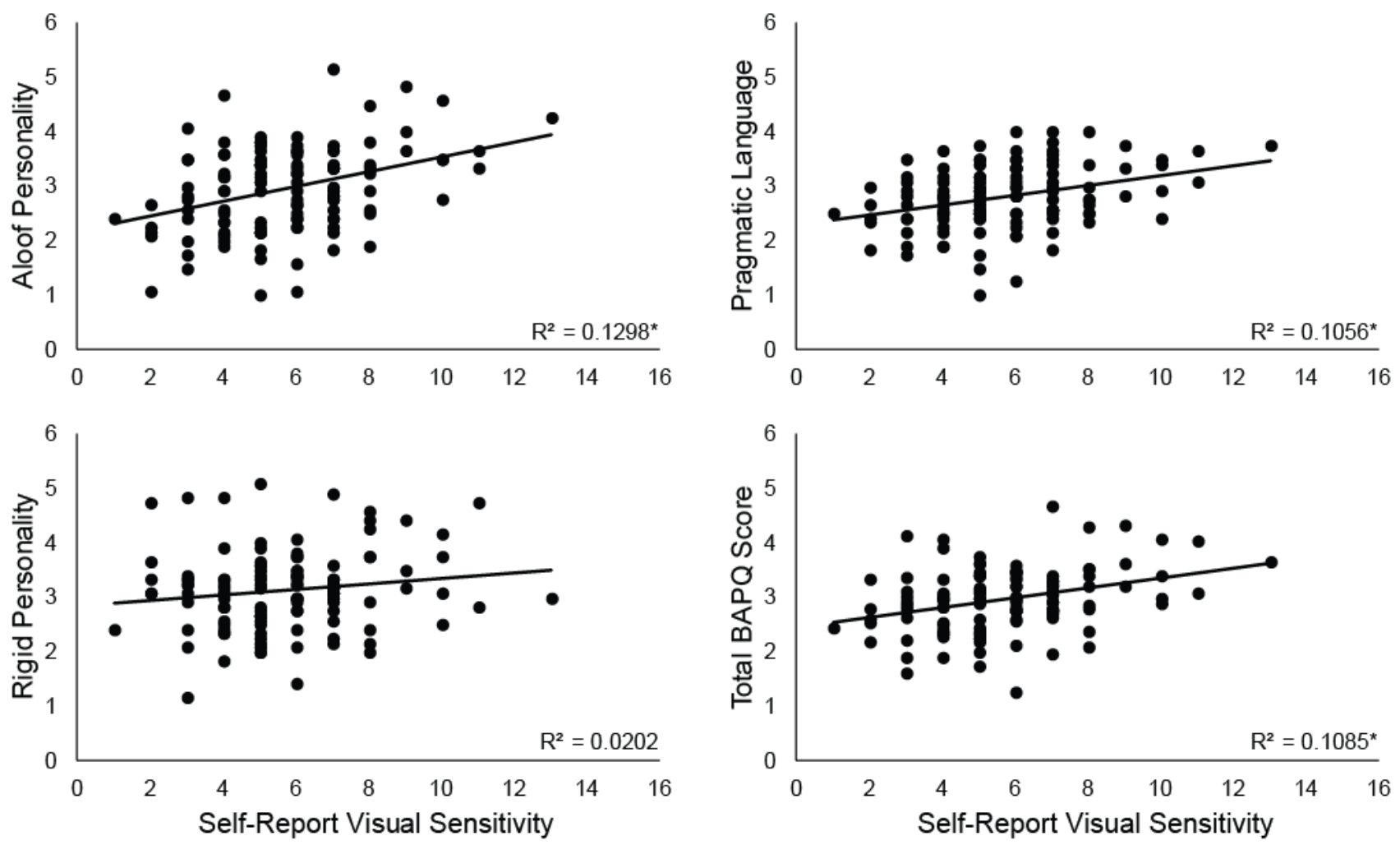

Figure 6: Correlations between self-report visual sensitivity and autistic traits.
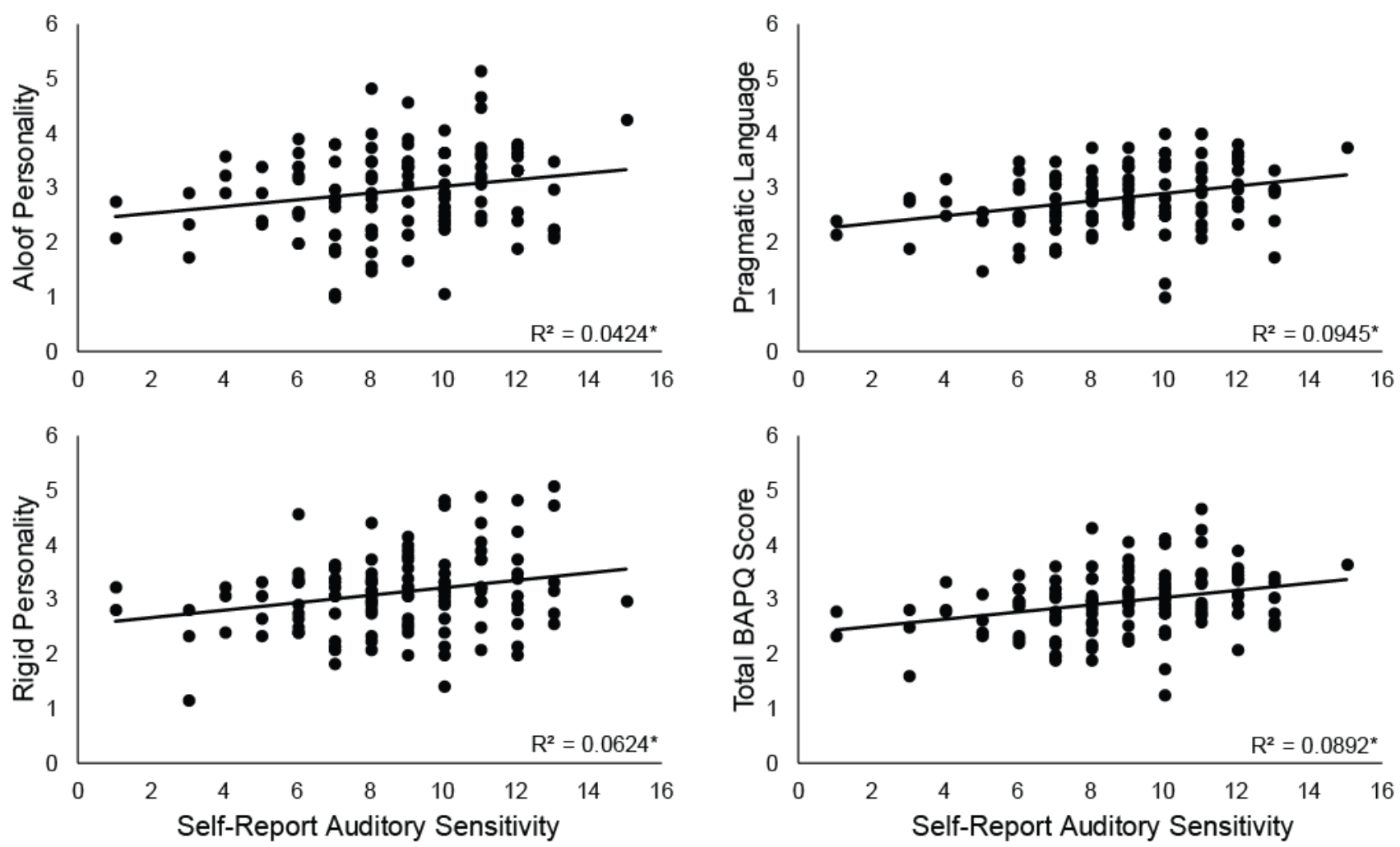

Figure 7: Correlations between self-report auditory sensitivity and autistic traits. 
Table 1: Predicting BAPQ subscales, Aloof Personality, Pragmatic Language, and Rigid

Personality, and Total BAPQ score, by visual thresholds and self-report visual sensitivity.

\begin{tabular}{|c|c|c|c|c|c|}
\hline Predictor & $\beta$ & t-value & $\begin{array}{c}\text { Zero-Order } \\
\text { Correlation (r) }\end{array}$ & $\begin{array}{c}\text { Partial } \\
\text { Correlation } \\
\text { (pr) }\end{array}$ & P-Value \\
\hline \multicolumn{6}{|c|}{ Model Predicting Aloof Personality: $R^{2}=0.202, F_{(2,104)}=13.169, p<0.001^{*}$} \\
\hline Visual Threshold & -0.301 & -3.410 & -0.255 & -0.317 & $<0.001^{*}$ \\
\hline $\begin{array}{l}\text { Self-report Visual } \\
\text { Sensitivity }\end{array}$ & 0.373 & 4.224 & 0.336 & 0.383 & $0.001^{*}$ \\
\hline \multicolumn{6}{|c|}{ Model Predicting Pragmatic Language: $R^{2}=0.100, F_{(2,104)}=5.758, p=0.004^{\star}$} \\
\hline Visual Threshold & -0.062 & -0.662 & -0.023 & -0.065 & 0.510 \\
\hline $\begin{array}{l}\text { Self-report Visual } \\
\text { Sensitivity }\end{array}$ & 0.317 & 3.384 & 0.310 & 0.315 & $0.001^{*}$ \\
\hline \multicolumn{6}{|c|}{ Model Predicting Rigid Personality: $R^{2}=0.062, F_{(2,104)}=3.423, p=0.036^{\star}$} \\
\hline Visual Threshold & -0.199 & -2.082 & -0.178 & -0.200 & $0.040^{\star}$ \\
\hline $\begin{array}{l}\text { Self-report Visual } \\
\text { Sensitivity }\end{array}$ & 0.175 & 1.828 & 0.151 & 0.176 & 0.070 \\
\hline \multicolumn{6}{|c|}{ Model Predicting Total BAPQ Score: $R^{2}=0.159, F_{(2,104)}=9.813, p<0.001^{*}$} \\
\hline Visual Threshold & -0.241 & -2.663 & -0.199 & -0.253 & $<0.001^{*}$ \\
\hline $\begin{array}{l}\text { Self-report Visual } \\
\text { Sensitivity }\end{array}$ & 0.348 & 3.840 & 0.318 & 0.352 & $0.009^{*}$ \\
\hline
\end{tabular}

Note: Asterisk indicated FDR-corrected statistical significance.

\section{Predicting Autistic Traits}

A series of multiple regressions were completed to ascertain whether autistic traits could be predicted by visual thresholds or self-report visual sensitivity. Given the predictor variables, visual thresholds and self-report visual sensitivity, Total BAPQ score $\left(F_{(2,104)}=\right.$ 9.813, $\left.p<0.001, R^{2}=0.159\right)$, Aloof Personality $\left(F_{(2,104)}=13.169, p<0.001, R^{2}=0.202\right)$, Pragmatic Language $\left(F_{(2,104)}=5.758, p=0.004, R^{2}=0.100\right)$, and Rigidity $\left(F_{(2,104)}=3.423, p\right.$ $=0.36, R^{2}=0.062$ ), were all significantly predicted. Visual thresholds were a significant predictor of the Aloof Personality and Rigidity subscales, and the Total BAPQ score, but not the Pragmatic Language subscale, whereas self-report visual sensitivity was a significant 
predictor of all the outcome measures except the Rigidity subscale, although it was trending towards significance (see Table 1 for detailed statistics). Visual sensitivity quantified through behavioural thresholds and self-report measures are predictive of autistic traits, broadly. Interestingly, these two different measurement approaches appear to be independently predictive of the outcome measures, again, suggesting that these two unique methods may actually assess separate constructs.

\section{Discussion}

The aim of this study was to compare assessment methods of sensory sensitivity and to relate those measures to behavioural traits displayed by clinical populations. This study examined whether behavioural thresholds and self-report sensitivity assessed independent constructs. Furthermore, as an example of how sensory processing issues have the potential to cascade into several higher-order, behavioural domains, each of these constructs was also related to autistic traits. A number of important findings were apparent within the data. First, results indicated that behavioural thresholds and self-report sensitivity were not related to each other and are indeed two distinct constructs that should be conceptually distinguished. Second, both behavioural thresholds and self-report sensitivity were related to autistic traits, however, the patterns of relationships with autistic traits observed, depended on the assessment type. Given the high prevalence of sensory issues across a range of neurodevelopmental disorders and other clinical populations, the implications of sensory processing issues in relation to higher-order behavioural symptoms may be far reaching across the population.

Sensory sensitivity refers to an individual's ability to detect a sensory signal or input. Therefore, we used a simple detection task to determine which individuals were more sensitive to sensory stimuli by demonstrating who was able to detect fainter stimuli in both the 
auditory and visual domains. This behavioural paradigm utilized an adaptive staircase to determine the level of stimulus intensity that was just noticeable to each individual participant, or the level at which each participant could detect that stimulus fifty percent of the time. This assessment resulted in a behavioural measurement of each individual's perceptual ability, or their sensitivity to both auditory and visual stimuli.

Sensory reactivity, on the other hand, refers to the intensity of the response in proportion to the intensity of the sensory stimulus. These are more likely to be overt behavioural reactions that are more easily captured through questionnaire data. Therefore, we assessed sensory reactivity through the AASP questionnaire measure of sensory processing. Sensory sensitivity and sensory reactivity are two distinct constructs and it is crucial to distinguish between them as they may result in the same overt behaviours even though the underlying mechanism resulting in said behaviour is different. For example, a fire alarm or other loud auditory stimulus is likely to be perceived as loud by most people and will likely result in an overt behavioural reaction in most people, such as flinching or covering of the ears. Alternatively, a microwave, or another such relatively quiet stimulus, will likely not elicit an overt behavioural reaction in typically-developed individuals, but may in clinical populations with sensory sensitivities like ASD for two distinct reasons. First, autistic individuals may perceive the microwave as being louder than their peers do (hypersensitivity), and thus an overt behavioural response would be expected, as it would be for a fire alarm. Second, autistic individuals may not perceive the microwave as louder than their TD peers, but still overtly respond (hyperreactive). Thus, both of these underlying mechanisms, sensory sensitivity and sensory reactivity, could result in the same behavioural response despite the response stemming from differing perceptions of that stimulus. Most importantly, this overt 
behaviour is captured in parent-report questionnaires, with no insight into the perceptual experience.

The results of the current investigation support this distinction between sensory sensitivity and sensory reactivity, as our behavioural and questionnaire measures were not related. If these two measures were assessing the same construct, we would expect the two measures to correlate, implying convergent validity. As hypothesized, broad relationships with autistic traits were found between sensitivity as measured through behavioural assessments and reactivity as measured through questionnaires. Furthermore, both sensory sensitivity and sensory reactivity were predictive of autistic traits, and in most cases displayed strong partial correlations suggesting that both constructs accounted for part of the variance in the equation. Overall, these results suggest that sensory sensitivity and sensory reactivity are distinct constructs that are both strongly implicated in the display of restricted interests, repetitive behaviours, adherence to routine, and difficulties with social communication and interaction. While these are the defining features of ASD, these higher-order behavioural traits that we have shown to be related to sensory processing are observed across neurodevelopmental disorders and many mental health disorders.

The analyses of autistic traits provide an example of the differential impacts sensory reactivity and sensory sensitivity have on clinically-related symptoms/traits. Our results revealed a relationship between sensory sensitivity and traits of both aloofness and rigidity, as well as overall autistic traits, such that increases in sensitivity were strongly related to increases in these traits. Specifically, these results indicated that greater visual sensitivity is related to greater instances of disinterest in social interactions and insistence on sameness which could provide evidence for the Intense World Syndrome, which argues that sensory overload may be an explanation for avoidance of social and novel situations. The Intense 
World Syndrome suggests that individuals with ASD are hyper-perceptive, hyper-attentive, and have hyper-memory due to excessive neuronal processing (Markram, Rinaldi, \& Markram, 2007). Therefore, individuals with greater sensitivity may avoid social situations because of the overstimulation resulting from a simple conversation. Likewise, novel situations and experiences are avoided by people who are hypersensitive because of the sensory overload associated with novelty, resulting in an insistence on sameness and rigidity of routine. This theory specifically explains the symptoms of ASD in relation to hypersensitivity, however, future research should aim to extend the support of the Intense World Syndrome explanation to other neurodevelopmental disorders with sensory sensitivities.

Although visual thresholds were strongly correlated with the behavioural traits of interest, the same was not true for auditory thresholds, for which we found no correlations with the BAPQ scores. While we hypothesized that auditory thresholds would be highly related to higher-order behavioural traits associated with ASD, there are several explanations for why there are differences between sensory domains in regard to their relationships with these traits. First, constant white noise was used to control for background noise in the current task and previous research has displayed greater difficulty detecting auditory stimuli when background noise is present (Green \& Sewall, 1962; Rawool, 2016), furthermore, research has shown that autistic individuals struggle with external noise processing (Park, Schauder, Zhang, Bennetto, \& Tadin, 2017) and have greater difficulty processing speech in background noise (Alcántara, Weisblatt, Moore, \& Bolton, 2004), which may extend to simple stimuli as well. To our knowledge, an equivalent study in the visual domain does not exist. Therefore, the increases in auditory sensitivity that were expected in individuals with greater autistic traits could have been hampered by the addition of background noise. Second, further 
research has suggested that individuals who are sensitive to auditory stimuli have greater difficulty successfully completing auditory discrimination tasks due to the aversive stimuli often used in these tasks (Jones et al., 2009). Again, suggesting that the individuals that we expected to perform better on this task due to their higher autistic traits, may have been subjected to confounding variables.

Visual reactivity was positively related to aloof personality traits, pragmatic language use, and overall autistic traits, but not rigidity, whereas auditory reactivity was positivity related to all aspects of autistic traits. These findings imply that individuals who have strong reactions to sensory stimuli, in both the auditory and visual domains, are more likely to exhibit higher-order behavioural traits related to ASD. According to previous research (Schulz \& Stevenson, 2018, 2019), we expected visual reactivity to be related to rigidity, however, this finding may suggest that sensitivity, rather than reactivity, plays a greater role in insistence on sameness in individuals with the broader autism phenotype and ASD.

Finally, we were able to predict all aspects of the broader autism phenotype, including both behavioural and social aspects, based on sensitivity and reactivity in the visual domain. Sensory sensitivity and sensory reactivity were both significant contributors, demonstrating that both reactivity and sensitivity are uniquely related to higher-order, behavioural traits associated with ASD. Importantly, results from the two questionnaires are more likely to be related to each other as opposed to correlating varying methods between questionnaires and behavioural thresholds. This bias speaks to the strong effect that sensory sensitivity as measured through behavioural assessments has on the prediction of autistic traits. These findings are important in the clinical realm, as they could have crucial implications on the treatment of sensory issues that are prevalent across neurodevelopmental disorders and possibly cascade into several behavioural domains, as demonstrated in this study. 
Although this study was conducted in a group of typically-developed adults, we predict that stronger associations would be found in autistic individuals and other clinical groups with sensory sensitivities. This prediction is founded in research on the broader autism phenotype which suggests that because autistic traits fall on the spectrum of ASD and the differences are quantitative in nature rather than qualitatively different (Landry \& Chouinard, 2016). However, further research is required to truly understand these relationships in populations with ASD and other neurodevelopmental disabilities.

These findings replicated and expanded the results in a previous study examining sensory sensitivity with a behavioural detection task and self-report sensitivity, in which both were predictive of autistic traits, specifically restrictive interests and repetitive behaviours, however the two sensory measures were indeed, unrelated (Schulz \& Stevenson, 2019). Our study used a similar detection task, however, the way in which we determined the behavioural threshold was modified. The previous study used the method of constant stimuli and determined the threshold level using psychometric functions whereas the current study utilized a staircase procedure that naturally narrowed in on the threshold of each participant. The benefit of using a staircase procedure is the increased specificity of stimuli that this procedure accommodates, facilitating a more accurate measure of sensory thresholds in a short period of time, which will allow for adaptation of this paradigm for children with neurodevelopmental disabilities. An additional difference was the questionnaire assessment used to encompass the full range of autistic traits, resulting in a greater understanding of ASD symptomatology as a whole and greater generalizability given the broad range of traits examined. Where the previous study used a specific measure of restricted interests and repetitive behaviours, namely, the Repetitive Behaviours Questionnaire, we broadened the research question to include autistic traits in general using the BAPQ. Finally, the current 
study expanded the study of sensory sensitivity to include auditory sensitivity in relation to autistic traits. Overall, both of these studies suggest that sensory sensitivity is strongly related to autistic traits, and more specifically, there are differences observed between sensory sensitivity and sensory reactivity. Therefore, through this study utilizing a modified behavioural paradigm and alternative questionnaires, we were successfully able to replicate the results of the previous study, suggesting these are robust findings, found across experimental paradigms.

In conclusion, sensory sensitivity and sensory reactivity are distinct constructs and need to be assessed as such. Aspects of this study replicated the findings of previous work using alternative methods suggests a robust relationship between sensitivity and higher-order behavioural traits and the clear distinction between sensory sensitivity and sensory reactivity. While the current study focused on autistic traits as an example of how sensory processing is related to behavioural symptoms, this work expanded the literature by including measures of auditory sensitivity and a broader range of traits associated with ASD, resulting in a greater generalizability of the findings to other disorders. Visual sensitivity, specifically, is highly implicated in the presence of higher-order behavioural traits associated with ASD in the general population and possibly contributes to a disinterest in social interactions and strict adherence to a rigid lifestyle. This study extended the literature by linking sensitivity with the social aspects of the autism phenotype. Further research should consider the role of both sensory sensitivity and sensory reactivity in the display of higher-order, behavioural traits across neurodevelopmental disorders and future literature should be careful in the distinction of these two constructs. 


\section{References}

Alcántara, J. I., Weisblatt, E. J., Moore, B. C., \& Bolton, P. F. (2004). Speech-in-noise perception in high-functioning individuals with autism or Asperger's syndrome. Journal of Child Psychology and Psychiatry, 45(6), 1107-1114.

Ashwin, E., Ashwin, C., Rhydderch, D., Howells, J., \& Baron-Cohen, S. (2009). Eagle-eyed visual acuity: an experimental investigation of enhanced perception in autism. Biological psychiatry, 65(1), 17-21.

Baranek, G. T., David, F. J., Poe, M. D., Stone, W. L., \& Watson, L. R. (2006). Sensory Experiences Questionnaire: discriminating sensory features in young children with autism, developmental delays, and typical development. Journal of Child Psychology and Psychiatry, 47(6), 591-601.

Baum, S. H., Stevenson, R. A., \& Wallace, M. T. (2015). Testing sensory and multisensory function in children with autism spectrum disorder. Journal of visualized experiments: JoVE(98).

Behrmann, M., Avidan, G., Leonard, G. L., Kimchi, R., Luna, B., Humphreys, K., \& Minshew, N. (2006). Configural processing in autism and its relationship to face processing. Neuropsychologia, 44(1), 110-129.

Benjamini, Y., \& Hochberg, Y. (1995). Controlling the false discovery rate: a practical and powerful approach to multiple testing. Journal of the royal statistical society. Series $B$ (Methodological), 289-300.

Bertone, A., Mottron, L., Jelenic, P., \& Faubert, J. (2003). Motion perception in autism: a "complex" issue. Journal of cognitive neuroscience, 15(2), 218-225.

Bertone, A., Mottron, L., Jelenic, P., \& Faubert, J. (2005). Enhanced and diminished visuospatial information processing in autism depends on stimulus complexity. Brain, 128(10), 2430-2441.

Blakemore, S.-J., Tavassoli, T., Calò, S., Thomas, R. M., Catmur, C., Frith, U., \& Haggard, P. (2006). Tactile sensitivity in Asperger syndrome. Brain and cognition, 61(1), 5-13.

Bonnel, A., McAdams, S., Smith, B., Berthiaume, C., Bertone, A., Ciocca, V., .. . Mottron, L. (2010). Enhanced pure-tone pitch discrimination among persons with autism but not Asperger syndrome. Neuropsychologia, 48(9), 2465-2475.

Bonnel, A., Mottron, L., Peretz, I., Trudel, M., Gallun, E., \& Bonnel, A.-M. (2003). Enhanced pitch sensitivity in individuals with autism: a signal detection analysis. Journal of cognitive neuroscience, 15(2), 226-235.

Brown, C., \& Dunn, W. (2002). Adolescent-adult sensory profile: user's manual: Therapy Skill Builders San Antonio.

Brown, C., Tollefson, N., Dunn, W., Cromwell, R., \& Filion, D. (2001). The adult sensory profile: Measuring patterns of sensory processing. American Journal of Occupational Therapy, 55(1), 75-82.

Caplette, L., Wicker, B., \& Gosselin, F. (2016). Atypical time course of object recognition in autism spectrum disorder. Scientific reports, 6, 35494.

Clery, H., Andersson, F., Bonnet-Brilhault, F., Philippe, A., Wicker, B., \& Gomot, M. (2013). fMRI investigation of visual change detection in adults with autism. Neurolmage: Clinical, 2, 303-312.

Crane, L., Goddard, L., \& Pring, L. (2009). Sensory processing in adults with autism spectrum disorders. Autism, 13(3), 215-228.

Cuthbert, B. N., \& Insel, T. R. (2013). Toward the future of psychiatric diagnosis: the seven pillars of RDoC. BMC medicine, 11(1), 126. 
De Jonge, M., Kemner, C., De Haan, E., Coppens, J., Van den Berg, T., \& Van Engeland, H. (2007). Visual information processing in high-functioning individuals with autism spectrum disorders and their parents. Neuropsychology, 21(1), 65.

Dunn, W. (1999). Sensory profile: User's manual San Antonio. TX: The Psychological Corporation.

Elliott, M. L., Romer, A., Knodt, A. R., \& Hariri, A. R. (2018). A connectome-wide functional signature of transdiagnostic risk for mental illness. Biological psychiatry, 84(6), 452459.

Engel-Yeger, B., Hardal-Nasser, R., \& Gal, E. (2011). Sensory processing dysfunctions as expressed among children with different severities of intellectual developmental disabilities. Research in Developmental Disabilities, 32(5), 1770-1775.

Ghanizadeh, A. (2011). Sensory processing problems in children with ADHD, a systematic review. Psychiatry Investigation, 8(2), 89-94.

Green, D. M., \& Sewall, S. T. (1962). Effects of background noise on auditory detection of noise bursts. The Journal of the Acoustical Society of America, 34(9A), 1207-1216.

Haesen, B., Boets, B., \& Wagemans, J. (2011). A review of behavioural and electrophysiological studies on auditory processing and speech perception in autism spectrum disorders. Research in autism spectrum disorders, 5(2), 701-714.

Haigh, S. M., Heeger, D. J., Heller, L. M., Gupta, A., Dinstein, I., Minshew, N. J., \& Behrmann, M. (2016). No difference in cross-modal attention or sensory discrimination thresholds in autism and matched controls. Vision research, 121, 85-94.

Houghton, D. C., Stein, D. J., \& Cortese, B. M. (2020). exteroceptive sensory abnormalities in childhood and adolescent anxiety and obsessive-compulsive disorder: a critical review. Journal of the American Academy of Child \& Adolescent Psychiatry, 59(1), 78-87.

Hrdlicka, M., Vodicka, J., Havlovicova, M., Urbanek, T., Blatny, M., \& Dudova, I. (2011). Brief report: significant differences in perceived odor pleasantness found in children with ASD. Journal of autism and developmental disorders, 41(4), 524-527.

Hurley, R. S., Losh, M., Parlier, M., Reznick, J. S., \& Piven, J. (2007). The broad autism phenotype questionnaire. Journal of autism and developmental disorders, 37(9), 16791690.

Insel, T., Cuthbert, B., Garvey, M., Heinssen, R., Pine, D. S., Quinn, K., ... Wang, P. (2010). Research domain criteria (RDoC): toward a new classification framework for research on mental disorders. In: Am Psychiatric Assoc.

Javitt, D. C. (2009). Sensory processing in schizophrenia: neither simple nor intact. Schizophrenia bulletin, 35(6), 1059-1064.

Jones, C. R., Happé, F., Baird, G., Simonoff, E., Marsden, A. J., Tregay, J., . . Charman, T. (2009). Auditory discrimination and auditory sensory behaviours in autism spectrum disorders. Neuropsychologia, 47(13), 2850-2858.

Järvinen-Pasley, A., Wallace, G. L., Ramus, F., Happé, F., \& Heaton, P. (2008). Enhanced perceptual processing of speech in autism. Developmental science, 11(1), 109-121.

Kern, J. K., Trivedi, M. H., Garver, C. R., Grannemann, B. D., Andrews, A. A., Savla, J. S., . . . Schroeder, J. L. (2006). The pattern of sensory processing abnormalities in autism. Autism, 10(5), 480-494.

Khalfa, S., Bruneau, N., Rogé, B., Georgieff, N., Veuillet, E., Adrien, J.-L., . . Collet, L. (2004). Increased perception of loudness in autism. Hearing research, 198(1-2), 87-92. Kinsbourne, M. (1980). Do Repetitive Movement Patterns in Children and Animals Serve a Dearousing Function? Journal of Developmental \& Behavioral Pediatrics, 1(1), 39-42. 
Kwakye, L. D., Foss-Feig, J. H., Cascio, C. J., Stone, W. L., \& Wallace, M. T. (2011). Altered auditory and multisensory temporal processing in autism spectrum disorders. Frontiers in integrative neuroscience, 4, 129.

Kéïta, L., Guy, J., Berthiaume, C., Mottron, L., \& Bertone, A. (2014). An early origin for detailed perception in Autism Spectrum Disorder: biased sensitivity for high-spatial frequency information. Scientific reports, 4, 5475.

Landry, O., \& Chouinard, P. A. (2016). Why we should study the broader autism phenotype in typically developing populations. Journal of Cognition and Development, 17(4), 584595.

Marco, E. J., Hinkley, L. B., Hill, S. S., \& Nagarajan, S. S. (2011). Sensory processing in autism: a review of neurophysiologic findings. Pediatr Res, 69(5 Pt 2), 48R-54R.

Markram, H., Rinaldi, T., \& Markram, K. (2007). The intense world syndrome-an alternative hypothesis for autism. Frontiers in neuroscience, 1, 6.

Mayer, J. L. (2017). The relationship between autistic traits and atypical sensory functioning in neurotypical and ASD adults: A spectrum approach. Journal of autism and developmental disorders, 47(2), 316-327.

McCleery, J. P., Allman, E., Carver, L. J., \& Dobkins, K. R. (2007). Abnormal magnocellular pathway visual processing in infants at risk for autism. Biological psychiatry, 62(9), 1007-1014.

Olsen, S. $\varnothing$. (1999). The relationship between the uncomfortable loudness level and the acoustic reflex threshold for pure tones in normally-hearing and impaired listeners-a meta-analysis. Audiology, 38(2), 61-68.

O'connor, K. (2012). Auditory processing in autism spectrum disorder: a review. Neuroscience \& Biobehavioral Reviews, 36(2), 836-854.

Park, W. J., Schauder, K. B., Zhang, R., Bennetto, L., \& Tadin, D. (2017). High internal noise and poor external noise filtering characterize perception in autism spectrum disorder. Scientific reports, 7(1), 1-12.

Piek, J. P., \& Dyck, M. J. (2004). Sensory-motor deficits in children with developmental coordination disorder, attention deficit hyperactivity disorder and autistic disorder. Human movement science, 23(3-4), 475-488.

Psychology Software Tools, I. (2016). E-Prime 3.

Rawool, V. W. (2016). Detection of auditory signals in quiet and noisy backgrounds while performing a visuo-spatial task. Noise \& health, 18(85), 283.

Robertson, A. E., \& Simmons, D. R. (2013). The relationship between sensory sensitivity and autistic traits in the general population. Journal of Autism and Developmental disorders, 43(4), 775-784.

Rogers, S. J., \& Ozonoff, S. (2005). Annotation: What do we know about sensory dysfunction in autism? A critical review of the empirical evidence. Journal of Child Psychology and Psychiatry, 46(12), 1255-1268.

Schulz, S. E., \& Stevenson, R. A. (2018). Sensory hypersensitivity predicts repetitive behaviours in autistic and typically-developing children. Autism, 1362361318774559.

Schulz, S. E., \& Stevenson, R. A. (2019). Differentiating between sensory sensitivity and sensory reactivity in relation to restricted interests and repetitive behaviours. Autism, 1362361319850402.

Serafini, G., Gonda, X., Canepa, G., Pompili, M., Rihmer, Z., Amore, M., \& Engel-Yeger, B. (2017). Extreme sensory processing patterns show a complex association with depression, and impulsivity, alexithymia, and hopelessness. Journal of affective disorders, 210, 249-257. 
Simmons, D. R., Robertson, A. E., McKay, L. S., Toal, E., McAleer, P., \& Pollick, F. E. (2009). Vision in autism spectrum disorders. Vision research, 49(22), 2705-2739.

Stewart, M. E., Griffiths, T. D., \& Grube, M. (2018). Autistic traits and enhanced perceptual representation of pitch and time. Journal of autism and developmental disorders, 48(4), 1350-1358.

Talay-Ongan, A., \& Wood, K. (2000). Unusual sensory sensitivities in autism: A possible crossroads. International Journal of Disability, Development and Education, 47(2), 201212.

Tavassoli, T., Latham, K., Bach, M., Dakin, S. C., \& Baron-Cohen, S. (2011). Psychophysical measures of visual acuity in autism spectrum conditions. Vision research, 51(15), 1778-1780.

Tobii. (2018). E-Prime Extensions.

Tomchek, S. D., \& Dunn, W. (2007). Sensory processing in children with and without autism: a comparative study using the short sensory profile. American Journal of occupational therapy, 61(2), 190-200.

Wallace, M. T., \& Stevenson, R. A. (2014). The construct of the multisensory temporal binding window and its dysregulation in developmental disabilities. Neuropsychologia, 64, 105123.

Watling, R. L., Deitz, J., \& White, O. (2001). Comparison of Sensory Profile scores of young children with and without autism spectrum disorders. American Journal of Occupational Therapy, 55(4), 416-423. 